\title{
Micro-Electro-Mechanical-Systems advances for measurements at small scales: the vertical silicon probes technology extended to near- field optics
}

\author{
B. Walter, E. Mairiaux, S. Eliet, J-.F. Lampin, and M. Faucher
}

\begin{abstract}
Instrumentation intending to go beyond the limits of current Atomic Force Microscope setups (AFM), and applications requiring AFM to be integrated in non-standards environmental conditions need sensors that overcome limitations given by available probes. We show that the vertical probe technology enabling AFM without laser is also applicable to scattering SNOM (Scanning Near Field Optical Microscopy).
\end{abstract}

\section{INTRODUCTION}

Atomic Force Microscopy is a commonly used technique to image surfaces at the nanoscale. Since the design of the first optical heads [1] and the emergence of batch fabricated cantilevers with integrated nanotips [2], a few decades of instrumental research led to the emergence of different families: general purpose machines upgradable with electrical measurements, AFMs dedicated to biology, AFMs optimized for optical techniques (Raman, SNOM), automated AFMs for metrology in semiconductor facilities, AFM for vacuum operation. The instrumental diversity mostly relies on probes using the cantilever design that has not significantly evolved. However, AFM instrumentation still faces numerous challenges, one being the design of compact heads suited to vacuum applications and extending the scanning capability to multiphysical sensing. In this context where laser-less tip detection is required, quartz probes are often used since the market does not provide other alternatives. Although these probes have a track record of impressive results [3],[4], these probes have been mainly used by the non-contact AFM community and it has been understood that miniaturization would be necessary to improve the signal to noise ratio [5]. Moreover, a monolithic tip integrated on the sensor and made with batch fabrication should be better for having probes with reproducible mechanical performances and moderate unity cost.

\section{PREVIOUS MEMS APPROACHES}

After the adoption of the silicon or silicon nitride cantilevers as the main probes used in volume by the AFM community, several attempts has been made to provide probes with integrated transducers. These works were based on MEMS technology and obtained results with different approaches: capacitive actuation [6], integration of

M.Faucher, J.-F. Lampin, and S. Eliet are with CNRS-IEMN, Institute of Electronics, Microelectronics and Nanotechnology, Villeneuve d'Ascq, France (corresponding author e-mail: marc.faucher@univ-lille.fr).

B. Walter and E. Mairiaux are with Vmicro SAS, cité scientifique, avenue Poincaré, Villeneuve d'Ascq, France. piezoresistive readout and/or piezoelectric actuation ([7], [8], [9], [10], [11], [12]), magnetic actuation [13]. MEMS technologies have also been applied to demonstrate sensors capable of parallel operation ([14],[15]) or the integration of the entire AFM scanning parts [16]. Membrane-based probes close to Capacitive Micromachined Ultrasonic Transducers (CMUTs) designs have also been proposed [17], [18], where detection still rely on optical means. In many mentioned works, tip motion is obtained out of the wafer plane; this implies to consider the constraint of tilting the tip holder. When electrical access is required by the transducers, the available space between the sensor and the surface to be scanned is small, imposing limitations of probe integration on the AFM head. Despite such work, a fully integrated sensor available in large volumes with a tip radius mastered below $30 \mathrm{~nm}$ has not emerged yet.

In previous work, we presented a probe design where a bulk mode resonator is used to provide an in-plane tip motion [19]. This concept that we refer as ring-probe makes use of capacitive transducers and was the first to permit the monolithic integration of a high frequency resonator with a silicon tip. It has enabled to reach $13.8 \mathrm{MHz}$, the highest vibrating frequency used in AFM imagery to date [20], [21],[22]. These ring probes made on $5 \mu \mathrm{m}$ thick Silicon-OnInsulator (SOI) have a stiffness (k) of $200 \mathrm{kN} / \mathrm{m}$ and enable tip amplitude typically below $0.1 \mathrm{~nm}$. Other detection approaches have been recently made were miniaturization offers perspectives for maintaining high frequencies with lower stiffness constant potentially under $10 \mathrm{kN} / \mathrm{m}$ [23]. Although the high k's of ring probes is an advantage for the AFM community interested in increasing the frequency and tackle ultra-high-speed imaging or spectroscopy, it also has limitations for many other communities looking at large amplitudes in Amplitude Modulation (AM) mode, quantitative imaging made in non-resonant modes, and SNOM.

\section{DESIGN AND FABRICATION OF VERTICAL PROBES FOR MULTIPURPOSE AFM}

Given the previous requirements and the need of integrated transducers able to operate with minimal interfacing, we proposed recently a technology for vertical probes [24]. The objectives are here to optimize the force gradient sensitivity instead of frequency increase, to obtain a tip with a high overhanging offset from the chip and having the capability of being electrically addressed. This put constraints on the design, since this implies to find a mode 
where the tip shape and mass can be varied while the mode existence and quality factor is not disturbed.

In order to investigate the applicability of the Vprobe technology to scattering scanning optical near-field microscopy (s-SNOM), we propose the design of Fig. 1. While the first Vprobes had no limitations related to frequency, the SNOM application requires a low frequency to maintain the optical signal harmonics in the detector bandwidth. Another motivation is to provide a probe not only suited to infrared but also to the THz range. Here, as in [25], the tip length should be close to the wavelength, typically $\sim 100 \mu \mathrm{m}$ at $2.5 \mathrm{THz}$. Moreover, the overhanging should be maximum in order to guarantee the highest solid angle for the incident laser and the scattered field. Thus, we have designed a large triangular shape at the end of the moving shuttle. The dimensions of the moving shuttle have been chosen after finite element modeling multiparameters optimization, targeting a pure vertical mode and repelling spurious mechanical modes far from the frequency zone of interest.

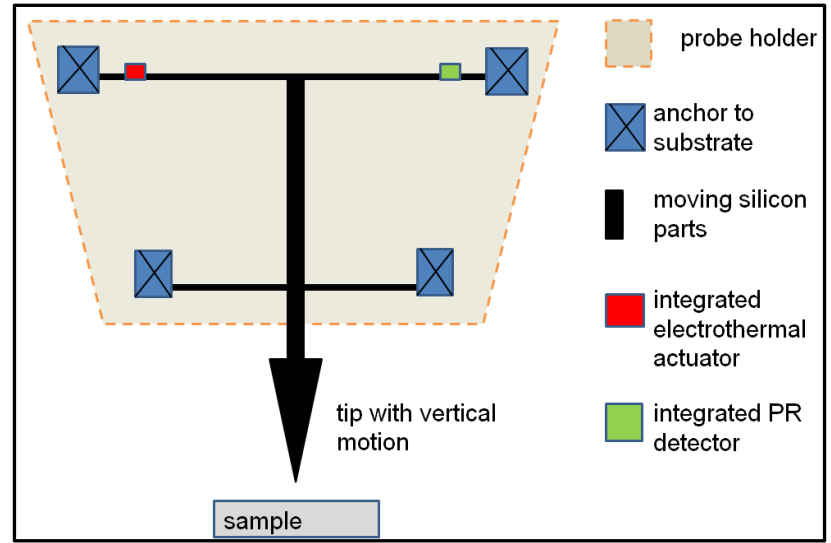

Figure 1: Principle of the Vprobes architecture.

The fabrication is summarized in Fig. 2: we make use of clean room processing steps that are typical in MEMS technologies and will be validated for lots production. We start from SOI wafers were the device layer has a $5 \mu \mathrm{m}$ thickness. After having defined alignment marks, the first step consists in patterning an implantation window, then exposing the transducers channels to boron $\left(70 \mathrm{keV} 6 \times 10^{13}\right.$ at.cm ${ }^{-2}$ followed by $20 \mathrm{keV} 6 \times 10^{13}$ at. $\mathrm{cm}^{-2}$ ). After Rapid thermal annealing, a second mask is generated, followed by a $\mathrm{Cl}_{2} / \mathrm{BCl}_{3}$ inductively coupled plasma etching. This step allows to define the resonator body, the main part of the tip that is triangle shaped, and one of the tip facets extending to the very end (see Figure 4). Then, low temperature oxide is deposited in order to form a sidewall along this facet and to protect the device elsewhere. In a third step, a lithography is performed to open a window in the oxide. This enables a starting surface for TMAH etching of silicon at step 4), which defines the nanotip. The next step 5) consists in removing this oxide by plasma etching and hydrofluoric acid. In step 6), aluminum is patterned by lift-off and ohmic contacts are formed to make the electrical wiring from transducers to metal lines and bonding pads. Then the chip holder is defined by bottom face lithography and deep reactive ion etching of the silicon handle wafer. Finally, vapor HF is used to release the shuttle and remove unwanted oxide residues.

Fig. 3 is a general view of the probe after the last fabrication steps and prior to detach the probe chips from the wafer. Fig. 4 is a SEM image highlighting the design key elements. In order to minimize the stiffness, we employed a needle-like design for the right flexural beams (c). The double $\mathrm{T}$ shape formed by the composite beams (c) and the shuttle (d), together with the large triangular tip (e) have been designed in order to reach an equivalent stiffness of $65 \mathrm{~N} / \mathrm{m}$ at $170 \mathrm{kHz}$ frequency for the in-plane mode of interest.

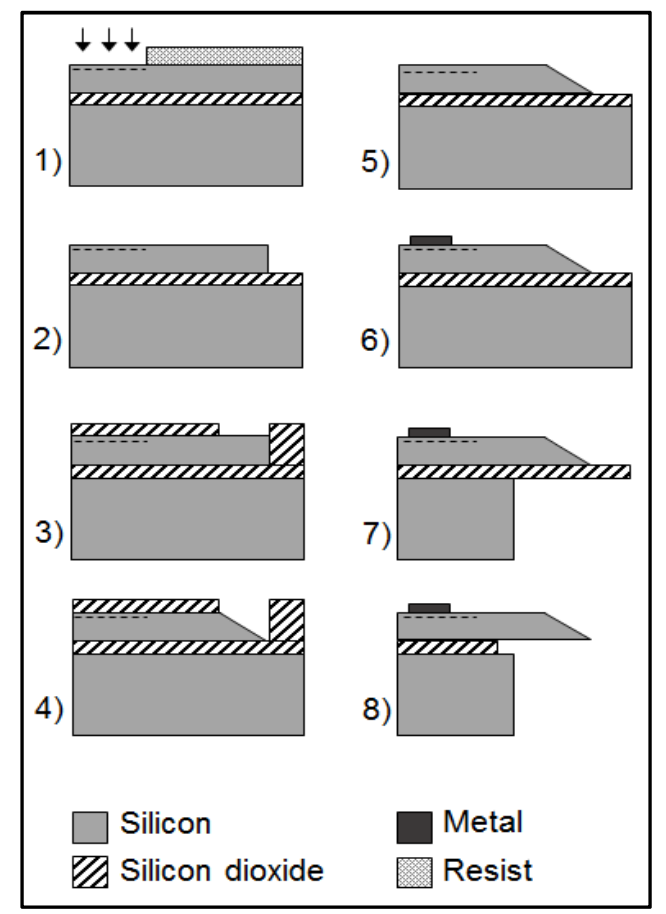

Figure 2: Main steps of the fabrication sequence for the Vprobes. The process starts from SOI substrates and enable full batch fabrication of probes with monolithically integrated nanotips, actuators, piezoresistive detectors, and resonant body.

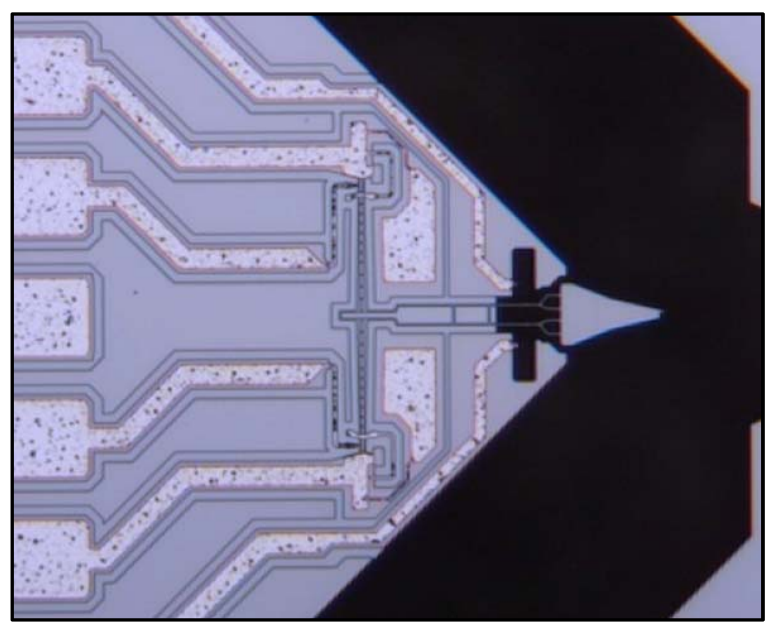

Figure 3: optical image of the large tip Vprobe prior chip detaching from the wafer. The device includes the chip holder, released silicon parts, metal lines going from tip and transducers to bonding pads located at left side. 


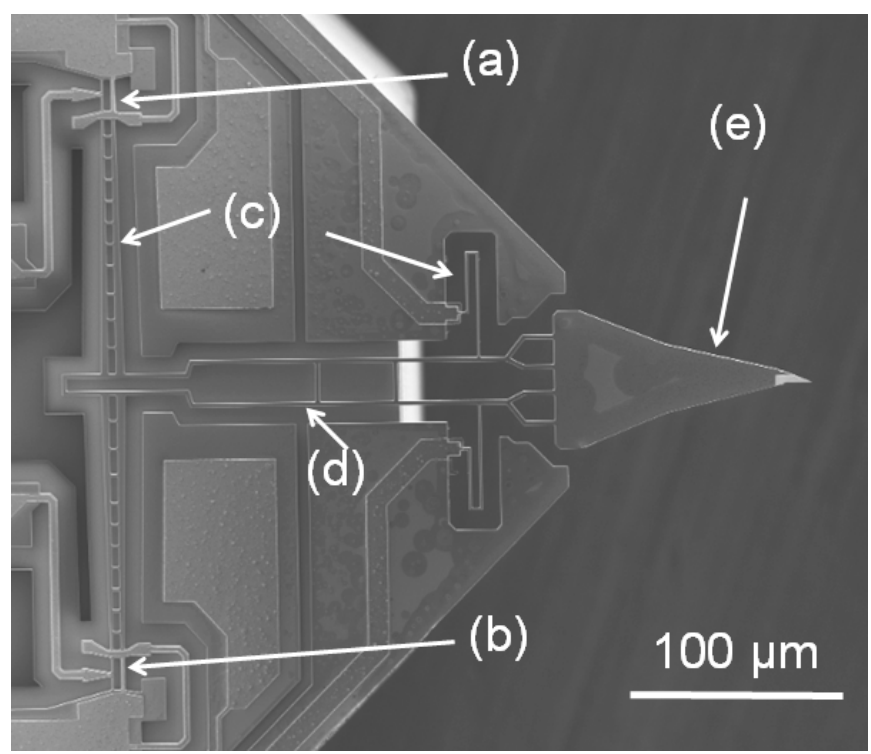

Figure 4: Scanning Electron Microscope Image of the probe featuring: (a) the piezoresistive transducer, (b) the electrothermal actuator, (c) the flexible beams, and (e) the large tip intending to offer a maximized solid angle for optical purposes.

\section{DEVICE CHARACTERIZATION}

Fig. 5 shows the electrical characterization of the probe behavior including the transducers. Here, the electrothermal actuator was biased with a $300 \mathrm{mV}$ DC voltage and $150 \mathrm{mV}$ $\mathrm{AC}$ voltage. The piezoresistive transducer was biased with a $300 \mathrm{mV} \mathrm{DC}$ voltage. A clear resonance is obtained at the expected frequency, with a large amplitude confirming the design advantages for efficient modulation of the scattered optical field as compared to quartz probes.

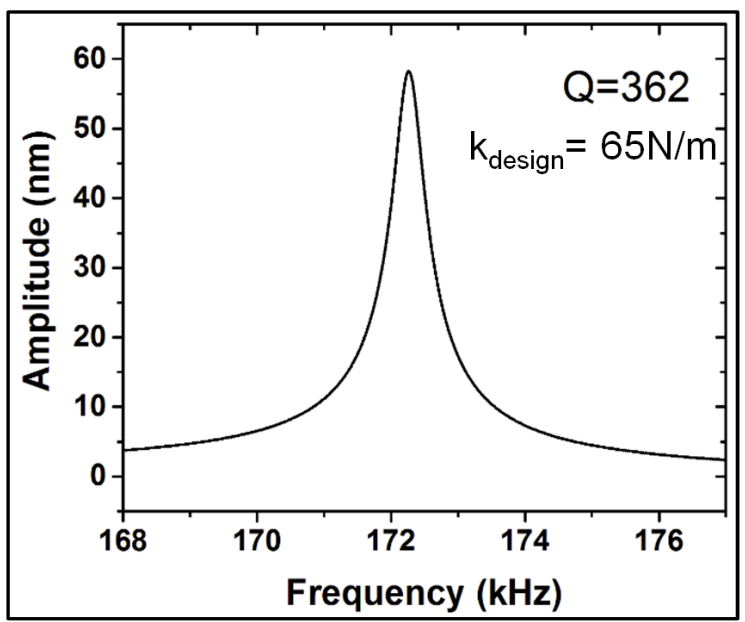

Figure 5: electrical characterization of the probe shown on fig.4.

In order to assess the possible elevation of tip temperature due to Joule heating by the transducers, we studied the temperature distribution on a Vprobe \#1 were the design has a smaller tip to transducers length (fig 2a of ref [24]). The electrothermal actuator has been biased with $500 \mathrm{mV}$ DC, 20 $\mathrm{mV} \mathrm{AC}$ and the detector with $100 \mathrm{mV} \mathrm{DC}$. For this configuration corresponding to $10 \mathrm{~nm}$ amplitude, calibrated separately by optical means, a weak hotspot is evidenced: the maximum recorded temperature elevation is $13^{\circ} \mathrm{C}$ at the transducer, whereas the tip has almost no measurable temperature elevation.

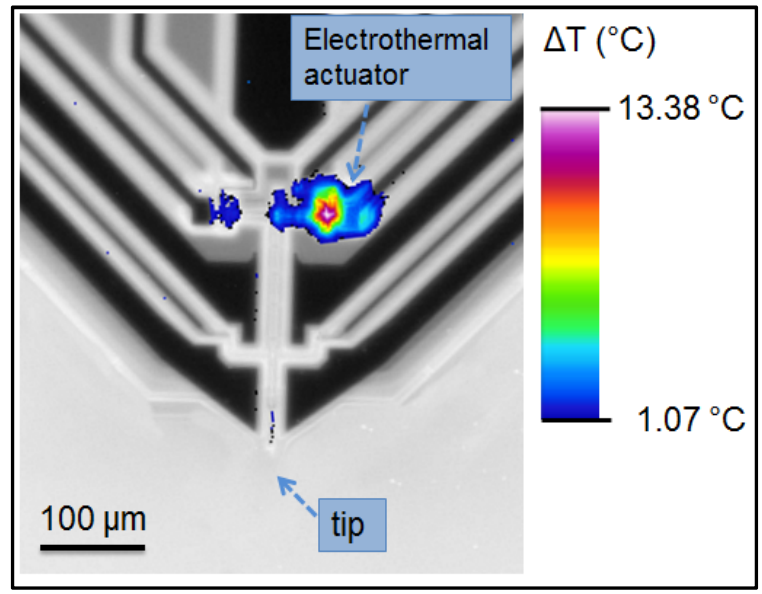

Figure 6: Thermal characterization on the Vprobe using an infrared camera. The electrothermal actuator provides a $10 \mathrm{~nm}$ amplitude, whereas the piezoresistive transducer is DC biased at 0.3 volts.

\section{AFM IMAGING WITH THE PROBE AND FIRST SNOM IMPLEMENTATION}

The new Vprobe design has been assessed in two steps. First, the probe was implemented on a modified commercial AFM. In this configuration shown on fig. 7, a Multimode AFM (Bruker) is used. In terms of mechanical aspects, the laser based optical head was removed and replaced with a metallic square holder. The electrical setup includes only 2 cables, an external amplifier, and 2 bias tees.

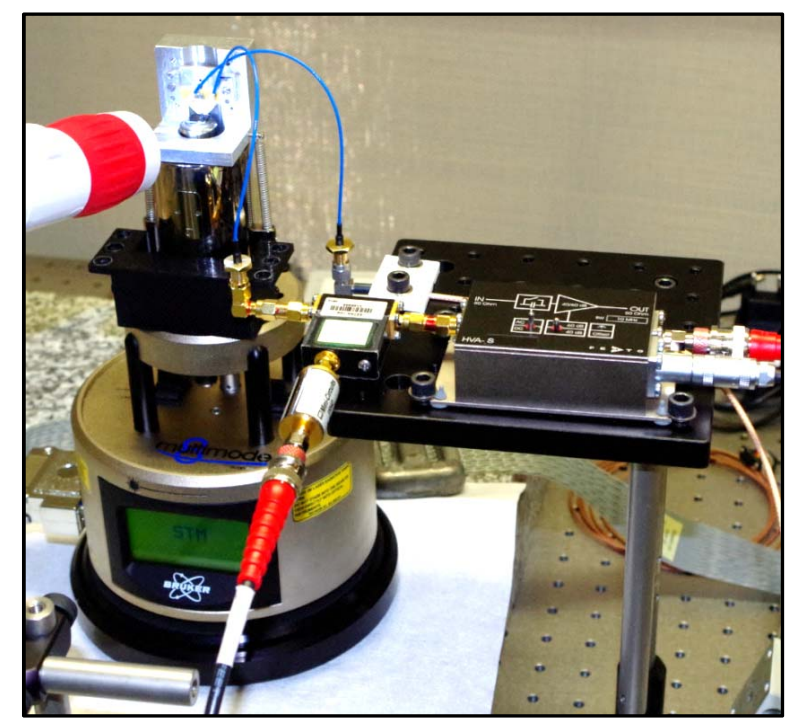

Figure 7: implementation of Vprobes in a commercial AFM for general purpose imaging.

The probe was operated in AM mode with a free amplitude of $20 \mathrm{~nm}$, a setpoint at $96 \%$ and a piezoresistive 
current bias at $3 \mathrm{~mA}$. A test sample of polyolefin elastomer/ polystyrene deposited onto $\mathrm{Si}$ was imaged. As evidenced in Fig. 8, the probe was able to image the microstructures and to give nanometric resolution at any location of a large scan.

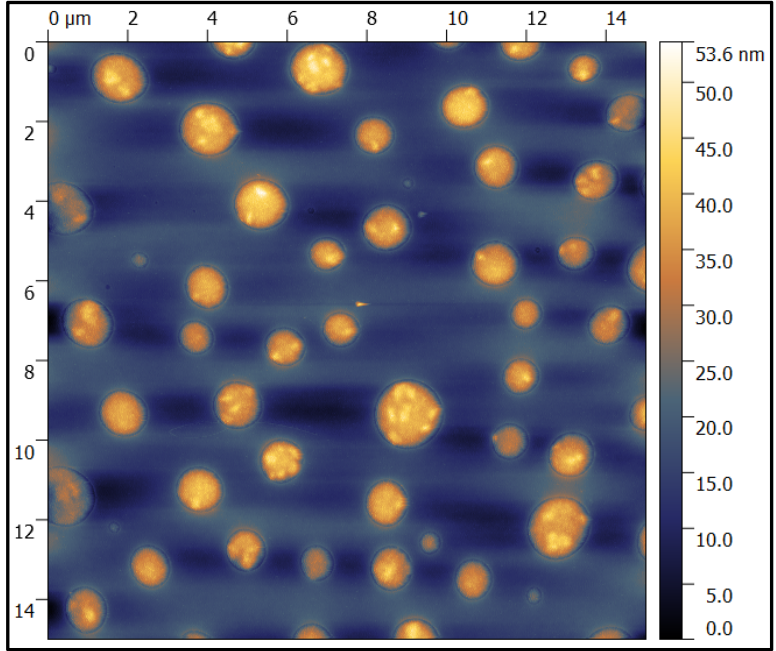

Figure 8: Assessement of the AFM capability of the Vprobe with large tip. the test sample consists of a mix of polyolefin elastomer and polystyrene (PS-LDPE-12M, Bruker).

In a second step, the probe was mounted onto a commercial s-SNOM (neaSNOM from Neaspec $\mathrm{GmbH}$ ) as shown on Fig. 9. Here, the actuator was fed by the signal intended to feed the piezoelectric ceramic, and the photodiode signal is replaced by the piezoresistive signal coming from the probe. Tip illumination was performed using a $\lambda=10.3 \mu \mathrm{m}$ quantum cascade laser source $\left(P_{\text {opt }}=3\right.$ $\mathrm{mW})$. The test sample consists of a graphene layer transferred on $\mathrm{SiO}_{2}$ and patterned [26]. Fig. 10 (b) shows the image given by the second harmonic lock-in demodulated signal. Harmonic demodulation is used to suppress the background [27]. Nanometric optical resolution $(<<\lambda)$ is obtained and correlated with the topography.

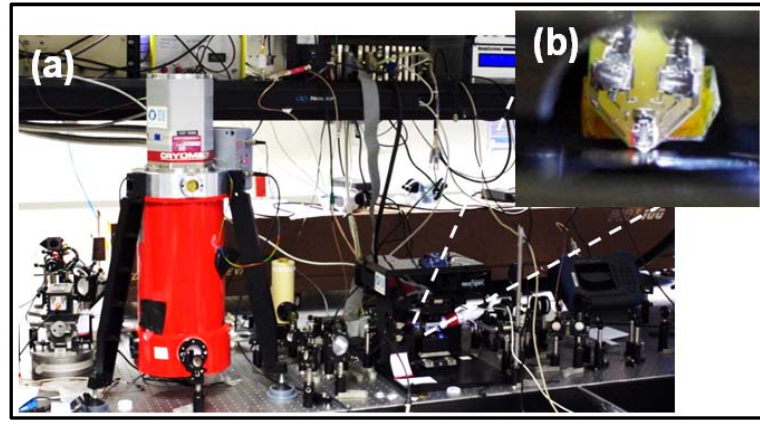

Figure 9: implementation of the last Vprobe version onto a commercial SNOM setup for IR and $\mathrm{THz}$ imaging. (a) The platform is based on a Neasnom microscope (Neaspec). (b) the Vprobe shown in fig. ?
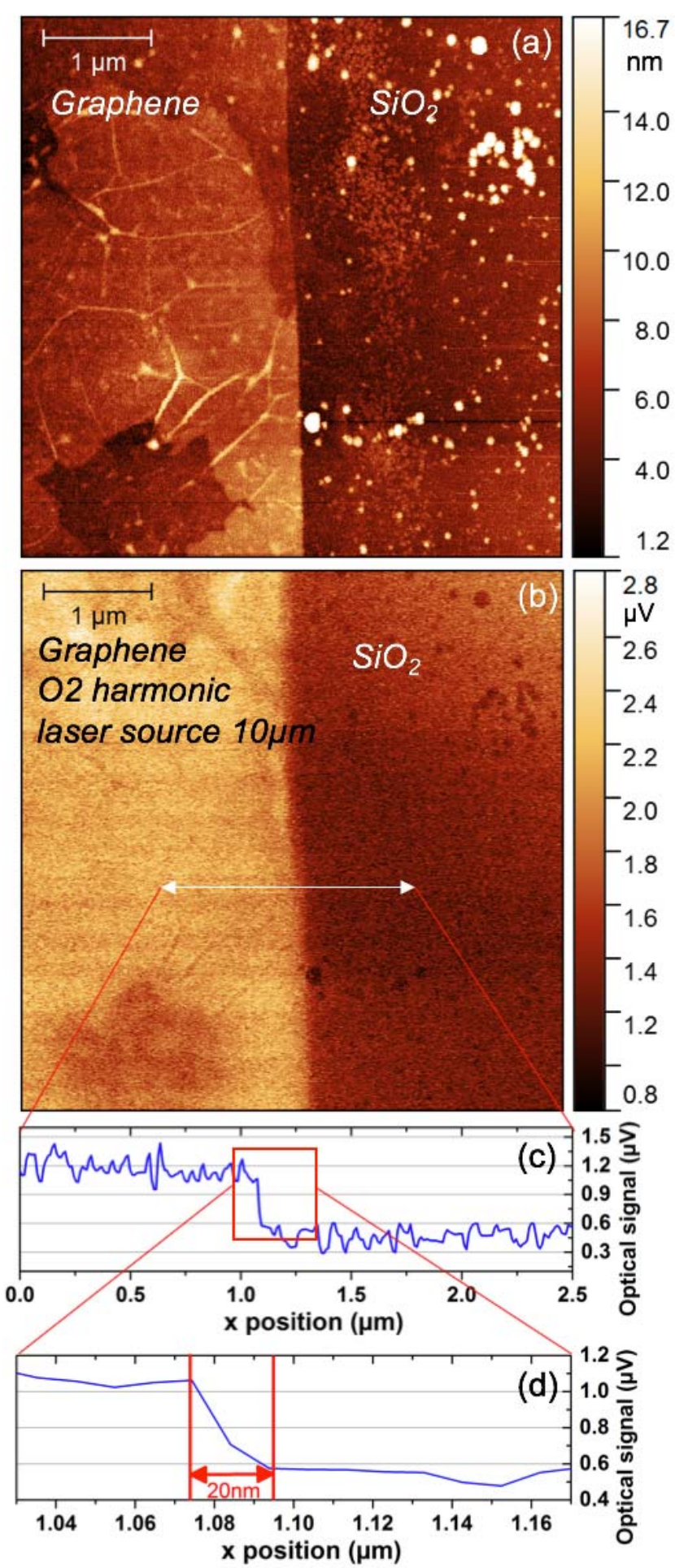

Figure 10: first s-SNOM image obtained with the Vprobe. (a) AFM topographic signal. (b) second harmonicoptical signal.(c) and (d) profile of the optical signal for assessing the nanoscale optical resolution 


\section{CONCLUSION}

The applicability of vertical probes (Vprobe) using fully integrated electrical transducers to s-SNOM imaging was demonstrated for the first time. In the future, we anticipate that the high tip length permitted by the MEMS design will enable to extend this approach to $\mathrm{THz}$ wavelengths $(\approx 100$ $\mu \mathrm{m})$, taking advantage of electromagnetic resonances engineering, and to extend this technique in other instrumental configurations.

\section{ACKNOWLEDGMENTS}

We acknowledge the equipex Excelsior ANR 11-EQPX0015 for funding part of this work.

\section{REFERENCES}

[1] G. Meyer and N. M. Amer, "Novel optical approach to atomic force microscopy," Applied Physics Letters, vol. 53, pp. 1045-1047, 1988.

[2] T. R. Albrecht, S. Akamine, T. E. Carver, and C. F. Quate, "Microfabrication of cantilever styli for the atomic force microscope," Journal of Vacuum Science \& Technology A: Vacuum, Surfaces, and Films, vol. 8, pp. 3386-3396, 1990.

[3] F. J. Giessibl, "Atomic resolution on $\mathrm{Si}(111)-(7 \times 7)$ by noncontact atomic force microscopy with a force sensor based on a quartz tuning fork," Applied Physics Letters, vol. 76, pp. 1470-1472, 2000.

[4] T. An, T. Nishio, T. Eguchi, M. Ono, A. Nomura, K. Akiyama, et al., "Atomically resolved imaging by low-temperature frequencymodulation atomic force microscopy using a quartz length-extension resonator," Review of Scientific Instruments, vol. 79, p. 033703, 2008.

[5] F. J. Giessibl, F. Pielmeier, T. Eguchi, T. An, and Y. Hasegawa, "Comparison of force sensors for atomic force microscopy based on quartz tuning forks and length-extensional resonators," Physical Review $B$, vol. 84 , p. $125409,09 / 06 / 2011$

[6] J. Brugger, N. Blamf, P. Renaudb, and N. F. D. Rooija, "Microlever with combined integrated sensor / actuator functions for scanning force microscopy," vol. 43, pp. 339-345, 1994.

[7] M. Tortonese, H. Yamada, R. C. Barrett, and C. F. Quate, "Atomic force microscopy using a piezoresistive cantilever," in Solid-State Sensors and Actuators, 1991. Digest of Technical Papers, TRANSDUCERS '91., 1991 International Conference on, 1991, pp. 448451.

[8] S. R. Manalis, S. C. Minne, and C. F. Quate, "Atomic force microscopy for high speed imaging using cantilevers with an integrated actuator and sensor," Applied Physics Letters, vol. 68, pp. 871-873, 1996/02/05 1996.

[9] B. W. Chui, T. D. Stowe, T. W. Kenny, H. J. Mamin, B. D. Terris, and D. Rugar, "Low-stiffness silicon cantilevers for thermal writing and piezoresistive readback with the atomic force microscope," Applied Physics Letters, vol. 69, pp. 2767-2769, 1996.

[10] R. P. Ried, H. J. Mamin, B. D. Terris, F. Long-Sheng, and D. Rugar, "6$\mathrm{MHz} 2-\mathrm{N} / \mathrm{m}$ piezoresistive atomic-force microscope cantilevers with INCISIVE tips," Journal of Microelectromechanical Systems, vol. 6, pp. 294-302, 1997.

[11]R. Boubekri, E. Cambril, L. Couraud, L. Bernardi, A. Madouri, M. Portail, et al., "Electrothermally driven high-frequency piezoresistive $\mathrm{SiC}$ cantilevers for dynamic atomic force microscopy Electrothermally driven high-frequency piezoresistive $\mathrm{SiC}$ cantilevers for dynamic atomic force microscopy," vol. 054304, pp. 0-9, 2014.

[12]M. Dukic, J. D. Adams, and G. E. Fantner, "Piezoresistive AFM cantilevers surpassing standard optical beam deflection in low noise topography imaging," Scientific Reports, vol. 5, p. 16393, 11/17/online 2015 .

[13]K. Nieradka, D. Kopiec, G. Małozięć, Z. Kowalska, P. Grabiec, P. Janus, et al., "Fabrication and characterization of electromagnetically actuated microcantilevers for biochemical sensing, parallel AFM and nanomanipulation," Microelectronic Engineering, vol. 98, pp. 676-679, 2012/10/01/2012.

[14] S. C. Minne, S. R. Manalis, and C. F. Quate, "Parallel atomic force microscopy using cantilevers with integrated piezoresistive sensors and integrated piezoelectric actuators," Applied Physics Letters, vol. 67, pp. 3918-3920, 1995.

[15]M. Despont, J. Brugger, U. Drechsler, U. Durig, W. Haberle, M. Lutwyche, et al., "VLSI-NEMS chip for AFM data storage," in Technical Digest. IEEE International MEMS 99 Conference. Twelfth IEEE International Conference on Micro Electro Mechanical Systems (Cat. No.99CH36291), 1999, pp. 564-569.

[16]N. Sarkar, G. Lee, and R. R. Mansour, "CMOS-MEMS dynamic FM atomic force microscope," in 2013 Transducers \& Eurosensors XXVII: The 17th International Conference on Solid-State Sensors, Actuators and Microsystems (TRANSDUCERS \& EUROSENSORS XXVII), 2013, pp. 916-919.

[17]F. L. Degertekin, "Novel Atomic Force Microscope Probes with Integrated Electrostatic Actuation and Optical Detection," in LEOS 2007 - IEEE Lasers and Electro-Optics Society Annual Meeting Conference Proceedings, 2007, pp. 832-833.

[18]H. Giray Oral, Z. Parlak, and F. Levent Degertekin, "Analysis of timeresolved interaction force mode AFM imaging using active and passive probes," Ultramicroscopy, vol. 120, pp. 56-63, 2012/09/01/ 2012.

[19]M. Faucher, B. Walter, A. S. Rollier, K. Seguini, B. Legrand, G. Couturier, et al., "Proposition of Atomic Force Probes Based on Silicon Ring-Resonators," in TRANSDUCERS 2007 - 2007 International SolidState Sensors, Actuators and Microsystems Conference, 2007, pp. 1529. 1532.

[20]E. Algré, B. Legrand, M. Faucher, B. Walter, and L. Buchaillot, "Surface microscopy with laserless MEMS based AFM probes," in 2010 IEEE 23rd International Conference on Micro Electro Mechanical Systems (MEMS), 2010, pp. 292-295.

[21]E. Algre, Z. Xiong, M. Faucher, B. Walter, L. Buchaillot, and B. Legrand, "MEMS Ring Resonators for Laserless AFM With SubnanoNewton Force Resolution," Journal of Microelectromechanical Systems, vol. 21, pp. 385-397, 2012.

[22]B. Legrand, J.-P. Salvetat, B. Walter, M. Faucher, D. Théron, and J.-P. Aimé, "Multi-MHz micro-electro-mechanical sensors for atomic force microscopy," Ultramicroscopy, vol. 175, pp. 46-57, 2017/04/01/ 2017.

[23]L. S. Pierre Etienne Allain, Colin Misner, Marc Gely, Estelle Mairiaux, Maxime Hermouet, Benjamin Walter, Giuseppe Leo, Sébastien Hentz, Marc Faucher, Guillaume Jourdan, Bernard Legrand, Ivan Favero, "Optomechanical Resonating Probe for Very High Speed Sensing of Atomic Forces," arXiv:1810.06209, 2018.

[24]B. Walter, E. Mairiaux, and M. Faucher, "Atomic force microscope based on vertical silicon probes," Applied Physics Letters, vol. 110, p. 243101, 2017.

[25]S. Mastel, M. B. Lundeberg, P. Alonso-González, Y. Gao, K Watanabe, T. Taniguchi, et al., "Terahertz Nanofocusing with Cantilevered Terahertz-Resonant Antenna Tips," Nano Letters, vol. 17, pp. 6526-6533, 2017/11/08 2017.

[26] A. Pagies, G. Deokar, D. Ducatteau, D. Vignaud, and J.-F. Lampin, "THz Near-field nanoscopy of graphene layers", Proceedings of the $40^{\text {th }}$ International Conference on Infrared, Millimeter, and Terahertz Waves (IRMMW-THz), Hong-Kong, China, 23-28 August 2015.

[27] R. Hillenbrand, T. Taubner, and F. Keilmann, "Phonon-enhanced lightmatter interaction at the nanometer scale", Nature, vol. 418, p. 159, 2002 . 